\title{
Representações gráficas procedimentais: elementos e relações
}

\author{
Procedural graphic representations: elements and \\ their relationships
}

análise gráfica,

Este artigo apresenta uma análise de representações gráficas buscando compreender representação, requisitos

\section{Diovana Mazur Damacena, Kelli C. A. Silva Smythe} a forma de estruturação e relacionamentos empregados nas composições. Para tanto, a partir de uma revisão bibliográfica, foi realizada a análise gráfica de dez representações de procedimentos. A análise teve como base variáveis sintáticas e semânticas provindas da teoria da sintaxe gráfica de Engelhardt (2002), contemplando representações de distintos níveis de complexidade. Os dados foram analisados quantitativamente, identificando elementos e as relações neste tipo de representação. Os resultados contribuíram para a identificação de requisitos úteis ao desenvolvimento de representações de procedimentos.

graphical analysis, representation, requirements

This paper presents an analysis of graphic representations aimed to understand the form of structuring and relationship used in the compositions. For this end, from a literature review, a graphic analysis of ten procedure representations was performed. The analysis was based on syntactic and semantic variables from Engelhardt's (2002) theory of graphic syntax, contemplating representations of different levels of complexity. The data was analyzed quantitatively, identifying elements and relationships used in this type of representation. The results contributed to identification of useful requirements for the development of procedural representations.

\section{Introdução}

No campo do Design a prática profissional estimula constantemente o designer a estar envolvido em equipes multidisciplinares, tanto na concepção quanto na implementação (BUENO; PADOVANI, 2015). Envolvendo profissionais de áreas distintas, os projetos de design necessitam que a estrutura do processo adotado seja compreendida por todos (SMYTHE; PRADO; SMYTHE-JR, 2016). Os projetos recebem um grande fluxo de informações a serem analisadas e gerenciadas sistematicamente, o que é complexo e pode ser um empecilho na execução dos mesmos (BARBosa et al., 2018). Assim, os métodos visuais e a visualização da informação apresentam-se como 
ferramentas para mitigar problemas no gerenciamento de tais projetos (BARBOSA et al., 2018).

Acrescenta-se que a utilização das representações gráficas, podem tangibilizar aspectos conceituais, elevando o entendimento sobre as etapas dos projetos, e, consequentemente, a assertividade da tecnologia desenvolvida (SMYTHE; PRADO; SMYTHE-JR, 2016). Além disso, as pessoas conseguem processar um grande volume de informação, a partir da visualização da informação, utilizando menor capacidade cognitiva (PATTERSON et al., 2014 apud BARBOSA et al., 2018).

Considerando o potencial das representações gráficas, este trabalho apresenta um estudo analítico, parte da pesquisa de iniciação científica da primeira autora, sobre elementos compositivos das representações procedimentais. Para tanto, a partir de uma revisão de literatura foi realizado um estudo analítico de representações procedimentais. Tal análise pautou-se na estrutura de sintaxe gráfica proposta por Engelhardt (2002), resultando na identificação das características gráficas elementares das representações, objetivando a geração de requisitos para o desenvolvimento futuro de representações de processos.

\section{Representações gráficas}

As transformações das representações gráficas desde a pré-história até hoje possibilitaram a facilitação da comunicação em seus diferentes contextos de uso. Tais representações podem ser definidas, segundo Engelhardt (2002), como artefatos visíveis em uma superfície mais ou menos plana, criadas com o objetivo de expressar informações. Complementarmente, Padovani (2012) destaca que o propósito das representações é comunicacional, ou seja, descrever, explicar, informar, instruir, o público alvo sem ambiguidades. Nesse sentido, Norman (1991) destaca as representações como artefatos cognitivos que, ao exibir ou operar informações artificialmente, auxiliam na compreensão da mensagem e, consequente realização da tarefa.

Norman (1993) pontua que a forma das representações dependem do contexto devendo, idealmente, almejar:

- Capturar características importantes e críticas do mundo representado, ignorando o irrelevante;

- Serem adequadas para a pessoa, potencializando o processo de interpretação;

- Serem adequadas para a tarefa, aumentando a capacidade de fazer julgamentos, para descobrir regularidades e estruturas relevantes.

No contexto de métodos e processos as representações gráficas podem ser de grande valia, uma vez que esses possuem estruturas que direcionam a um caminho, destacando atividades sequenciais 
com relacionamentos lógicos (GIL, 2008; LIMA, 2017). Deste modo, a explicitação visual tem potencial comunicativo positivo no auxílio da compreensão dos componentes, passos e suas relações.

\subsection{Sintaxe gráfica}

Diante da identificação da relevância de representações gráficas, torna-se importante ao design da informação compreender seus elementos e relações para transmissão de significado.

Nesse sentido, Engelhardt (2002), embasado na semiologia gráfica definida por Bertin (1967), propôs uma estrutura para análise da sintaxe e significado das representações. Para o autor, uma representação gráfica é um objeto gráfico (elementar ou composto), consistindo em:

- Um espaço gráfico que é ocupado por ele;

- Um conjunto de objetos gráficos, que estão contidos naquele espaço gráfico;

- Um conjunto de relações gráficas em que esses objetos gráficos estão envolvidos.

A Figura 1 apresenta a decomposição sintática de um objeto gráfico composto.

Partindo do relacionamento objetos/espaço gráfico, Engelhardt (2002) descreve variáveis, aqui apresentadas em Atributos visuais perceptíveis; Estruturas sintáticas: relações gráficas dos objetos; Interpretação das representações: análise semântica de uma representação gráfica, ocorrendo paralelamente à análise sintática

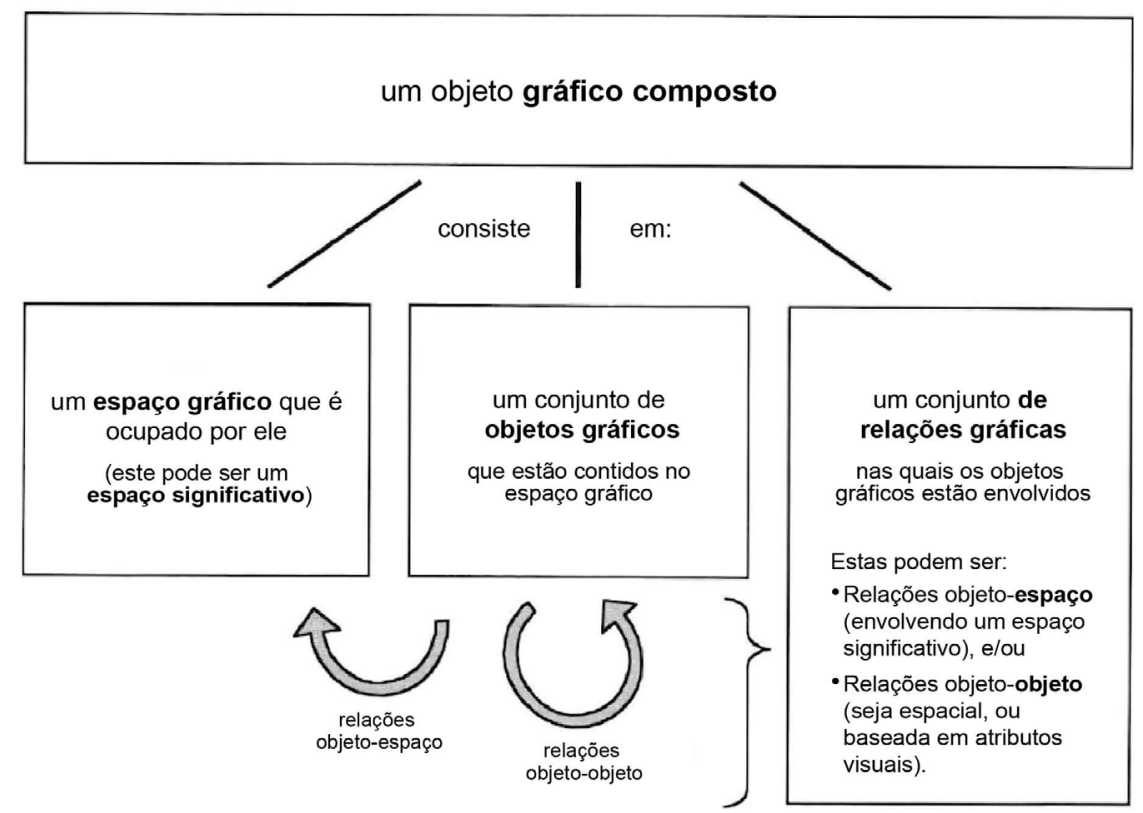

Figura 1 Decomposição sintática. Fonte: Adaptado de Engelhardt (2002). 
de sua estrutura, e; Classificação das representações: denominação das representações baseadas nos tipos de estrutura sintática e da informação expressa (Quadro 1).

Essa estrutura apresenta-se como auxiliadora na compreensão da composição de uma representação e relações dos objetos ali apresentados. Portanto, será aqui utilizada como subsídio para análise de representações gráficas utilizadas no contexto de apresentação de métodos e processos.

Quadro 1 Síntese categorizada das variáveis gráficas de Engelhardt (2002). Fonte: os autores.

\begin{tabular}{|c|c|c|}
\hline \multicolumn{3}{|l|}{ Atributos visuais perceptíveis } \\
\hline Espaciais: & \multicolumn{2}{|l|}{ Forma, Tamanho, Orientação, Posição. } \\
\hline Preenchimento: & \multicolumn{2}{|l|}{ Cor, Textura, Valor. } \\
\hline \multicolumn{3}{|l|}{ Estruturas sintáticas } \\
\hline \multirow{2}{*}{ Básicas: } & Relação objeto-objeto & $\begin{array}{l}\text { Agrupamento, Separação, Alinhamento, } \\
\text { Vinculação, Contenção, Sobreposição. }\end{array}$ \\
\hline & Relação objeto-espaço & $\begin{array}{l}\text { Estruturas espaciais não-ancoradas, } \\
\text { e estruturas espaciais ancoradas. }\end{array}$ \\
\hline Compostas: & $\begin{array}{l}\text { Combinação simultânea, } \\
\text { Aninhamento, Inserção de } \\
\text { Fundo, Múltiplo Gráfico. }\end{array}$ & \\
\hline \multicolumn{3}{|c|}{ Interpretação das representações gráficas } \\
\hline Tipos de correspondência: & \multicolumn{2}{|l|}{$\begin{array}{l}\text { Literal, Metafórica, } \\
\text { Convencionado-arbitrário, } \\
\text { Rebús, Metonímica. }\end{array}$} \\
\hline Modo de expressão: & \multicolumn{2}{|l|}{ Pictóricos e não-pictóricos. } \\
\hline Significado dos objetos gráficos: & \multicolumn{2}{|l|}{$\begin{array}{l}\text { Informativos, Referenciais, } \\
\text { Decorativos. }\end{array}$} \\
\hline \multicolumn{3}{|l|}{ Classificação das representações } \\
\hline Representações primárias: & \multicolumn{2}{|l|}{$\begin{array}{l}\text { Mapa, Figura, Gráfico estatístico, Gráfico } \\
\text { de tempo, Diagrama de vinculação, } \\
\text { Diagrama de agrupamento, Tabela, } \\
\text { Símbolo, Texto. }\end{array}$} \\
\hline Representações híbridas: & \multicolumn{2}{|l|}{$\begin{array}{l}\text { Mapa estatístico, Mapa de caminho, } \\
\text { Gráfico cronológico estatístico, } \\
\text { Diagrama cronológico de vinculação, } \\
\text { Diagrama estatístico de vinculação. }\end{array}$} \\
\hline
\end{tabular}

\section{Desenvolvimento metodológico}

Com o objetivo de evidenciar os elementos constituintes das representações gráficas de métodos e processos, realizou-se uma análise gráfica de representações a partir das variáveis propostas por Engelhardt (2002). 
Para tanto, utilizou-se uma amostra de dez representações, identificadas a partir de buscas em periódicos e websites. Os critérios para seleção da amostra foram: (a) representações de processos ou métodos; (b) diferentes graus de complexidade, e; (c) variedade dos elementos compositivos.

Os resultados foram analisados de forma quantitativa e descritiva, buscando identificar requisitos eficazes no desenvolvimento de representações. As representações analisadas podem ser visualizadas nas figuras a seguir.
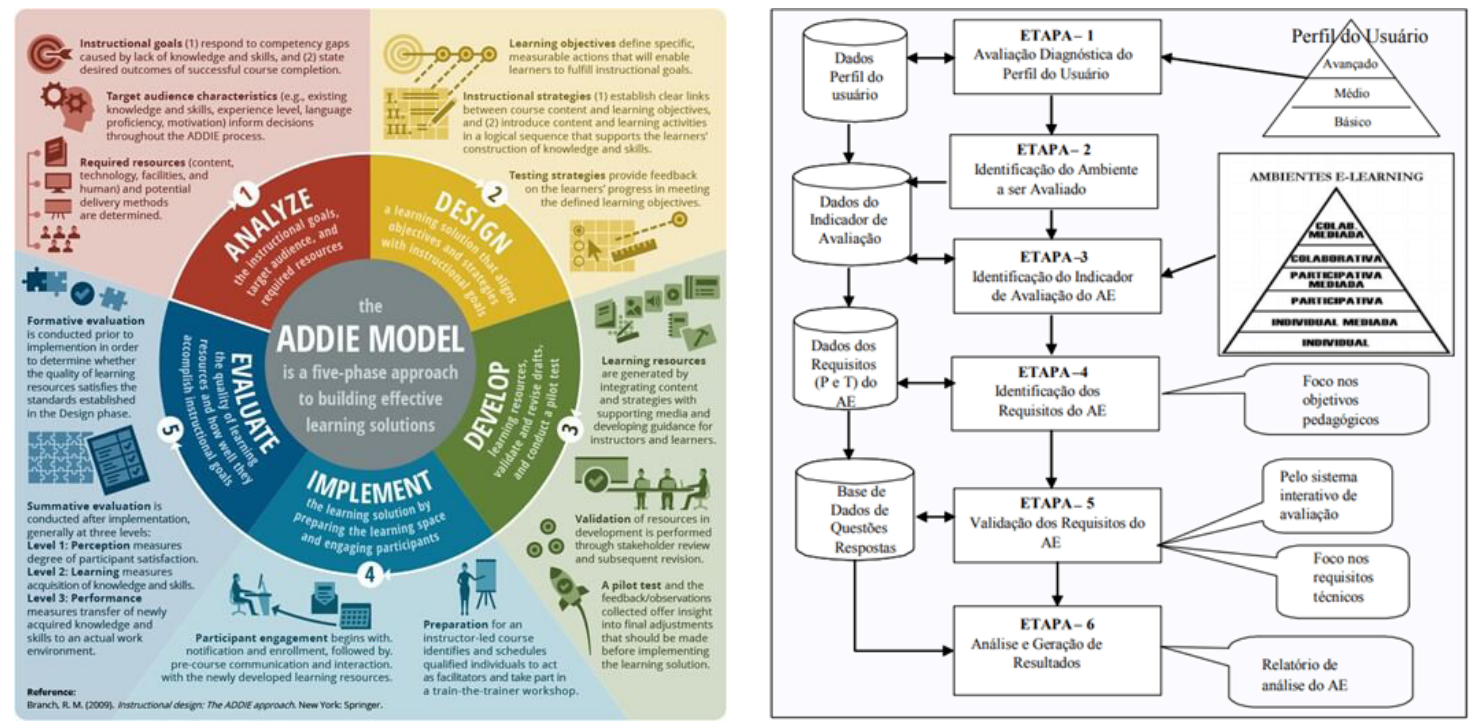

Figura 2 Representação 1 - Modelo ADDIE e 2 - MA-AE. Fonte: Obsidian Learning (2017) / Kemczinski (2005).
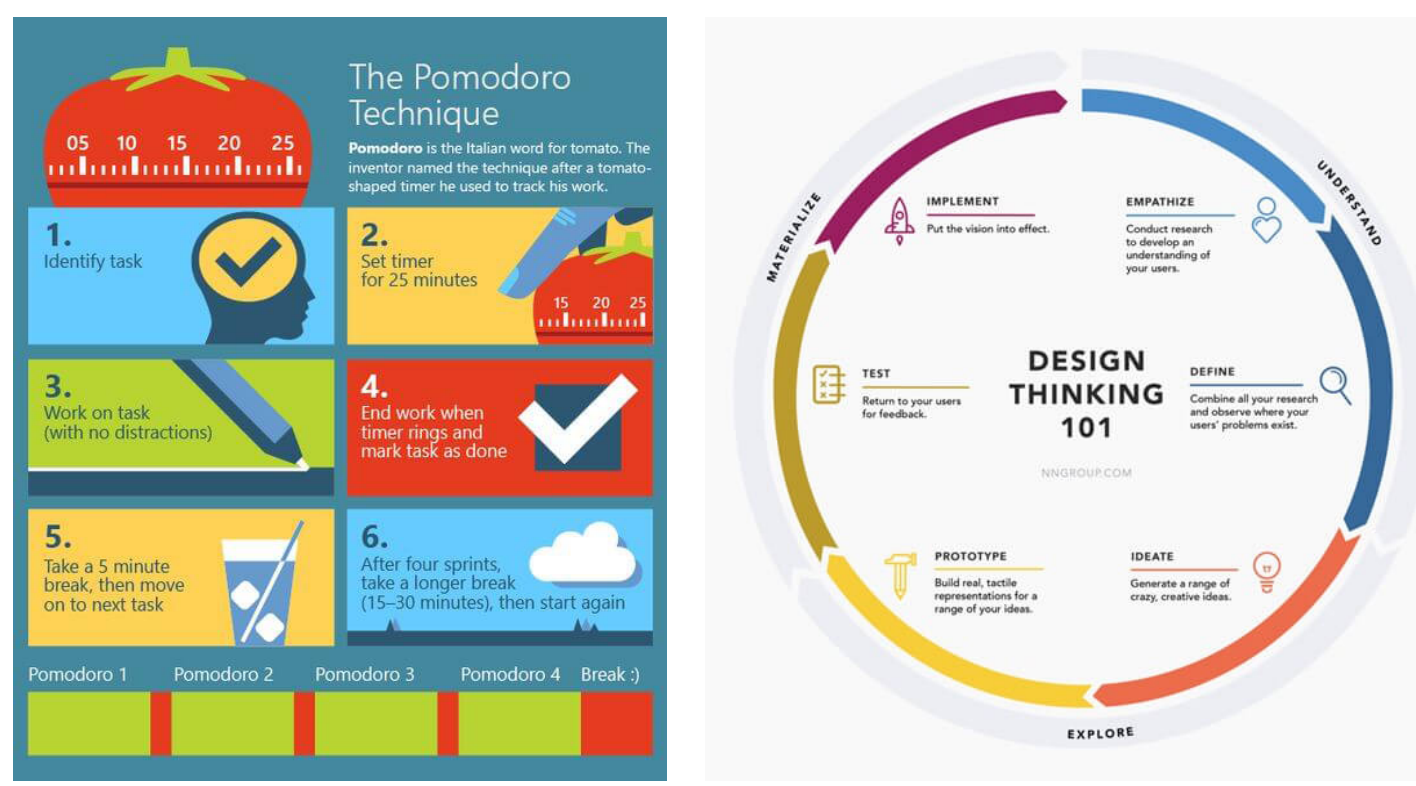

Figura 3 Representação 3 -Técnica Pomodoro e 4 - Processo Design Thinking. Fonte: Microsoft Partner Community (2017) / Octadesk (2017). 


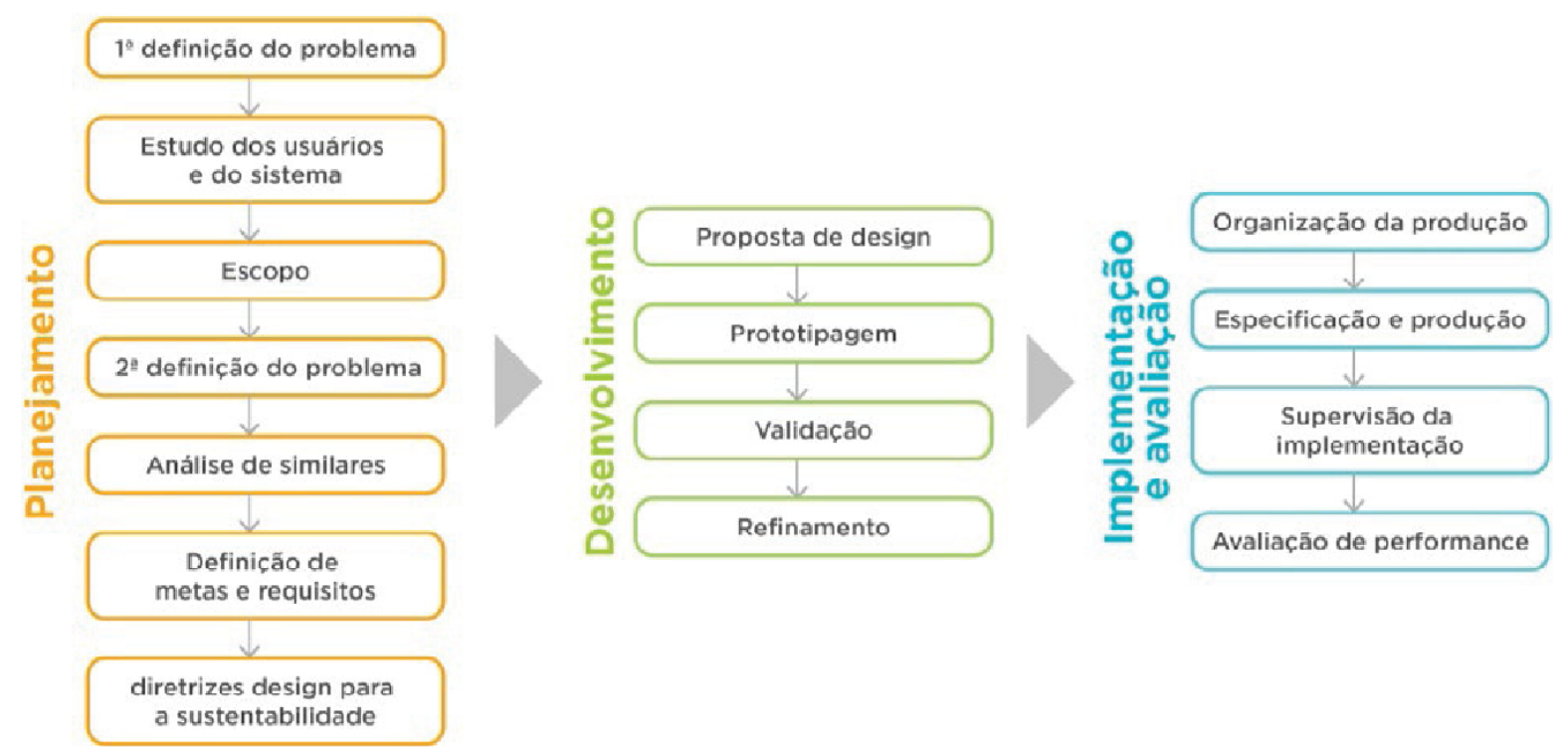

Figura 4 Representação 5 - Processo de wayfinding. Fonte: Smythe (2018).

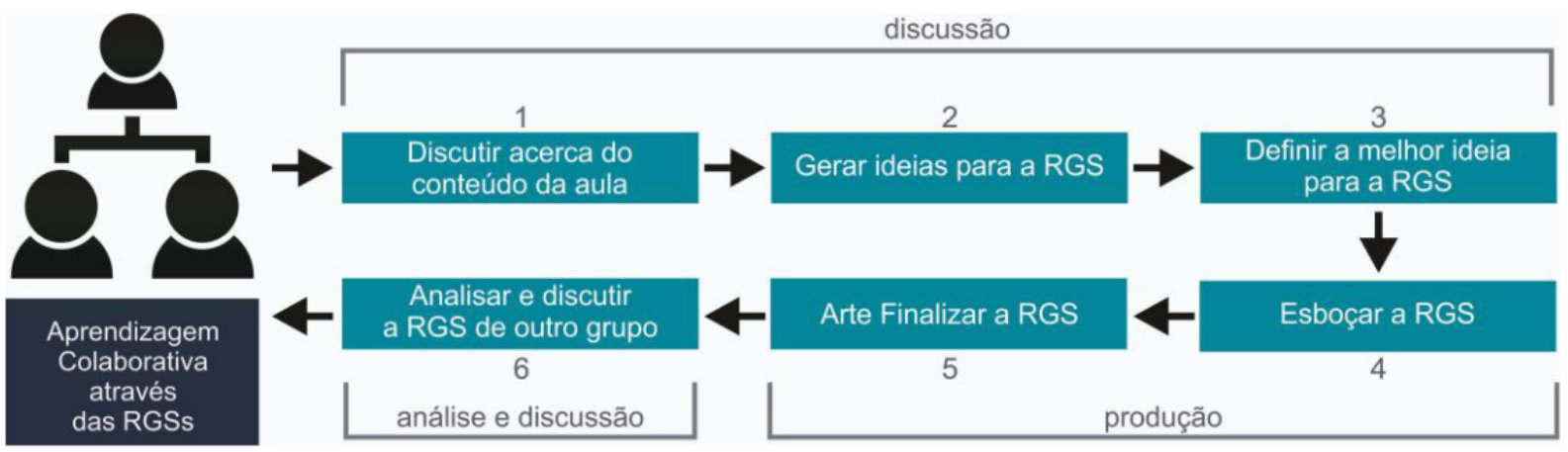

Figura 5 Representação 6 - Aprendizagem colaborativa. Fonte: Bueno; Padovani (2015).

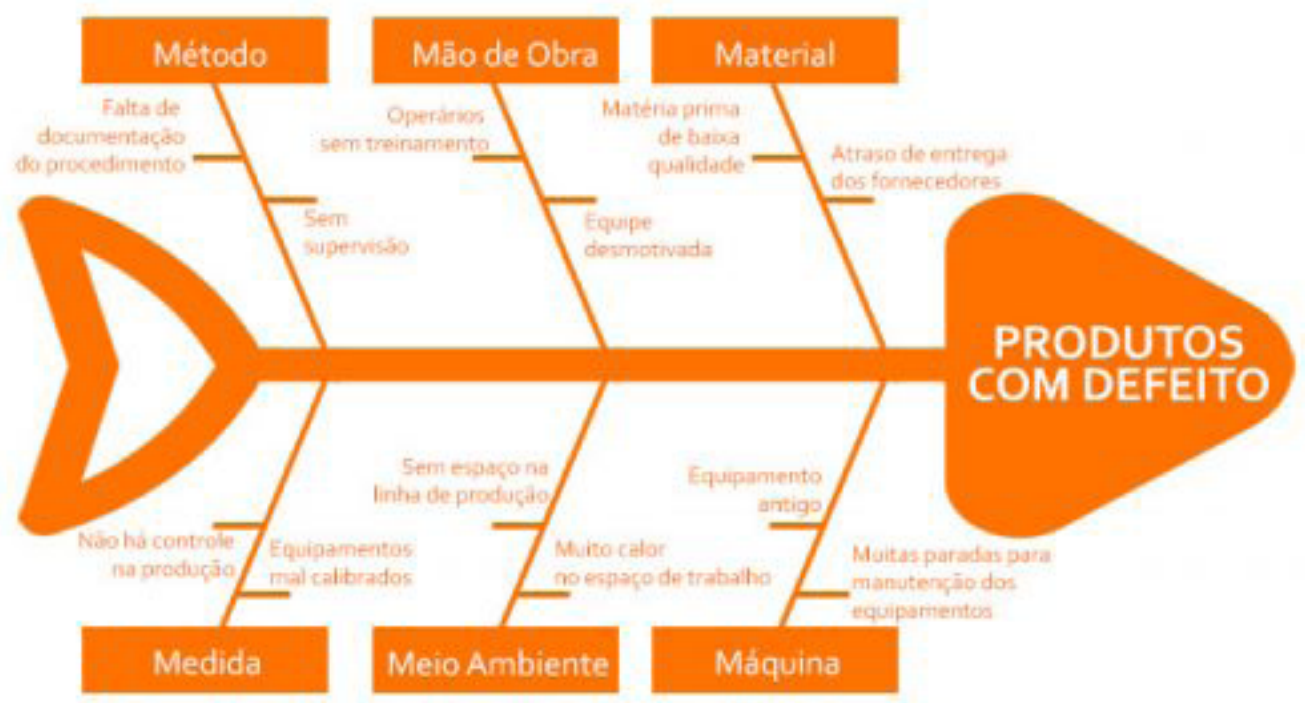

Figura 6 Representação 7 - Diagrama de Ishikawa. Fonte: Lean Blog (2018). 

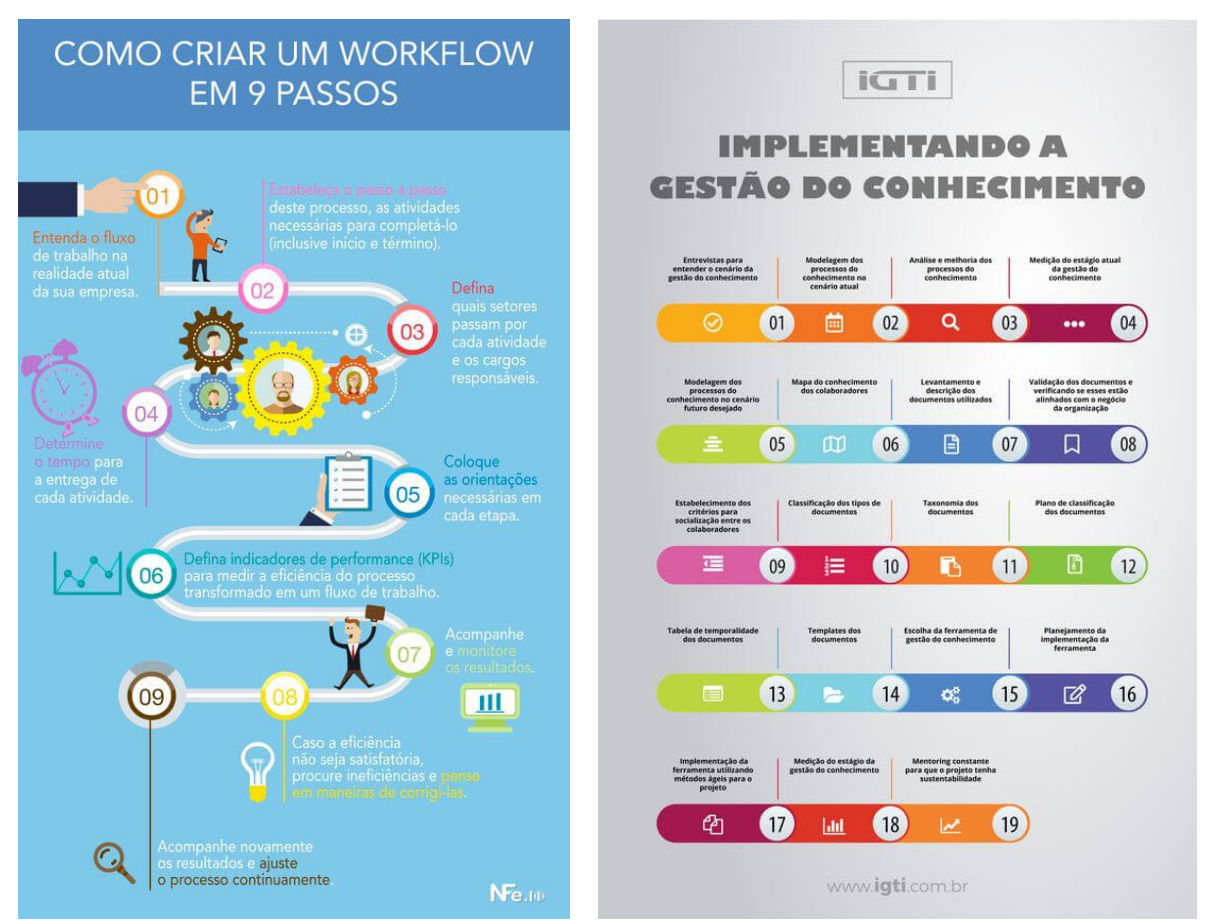

Figura 7 Representação 8 - Criação de Workflow e 9 - Implementação gestão do conhecimento. Fonte: NFE (2017) / IGTI Blog (2018).

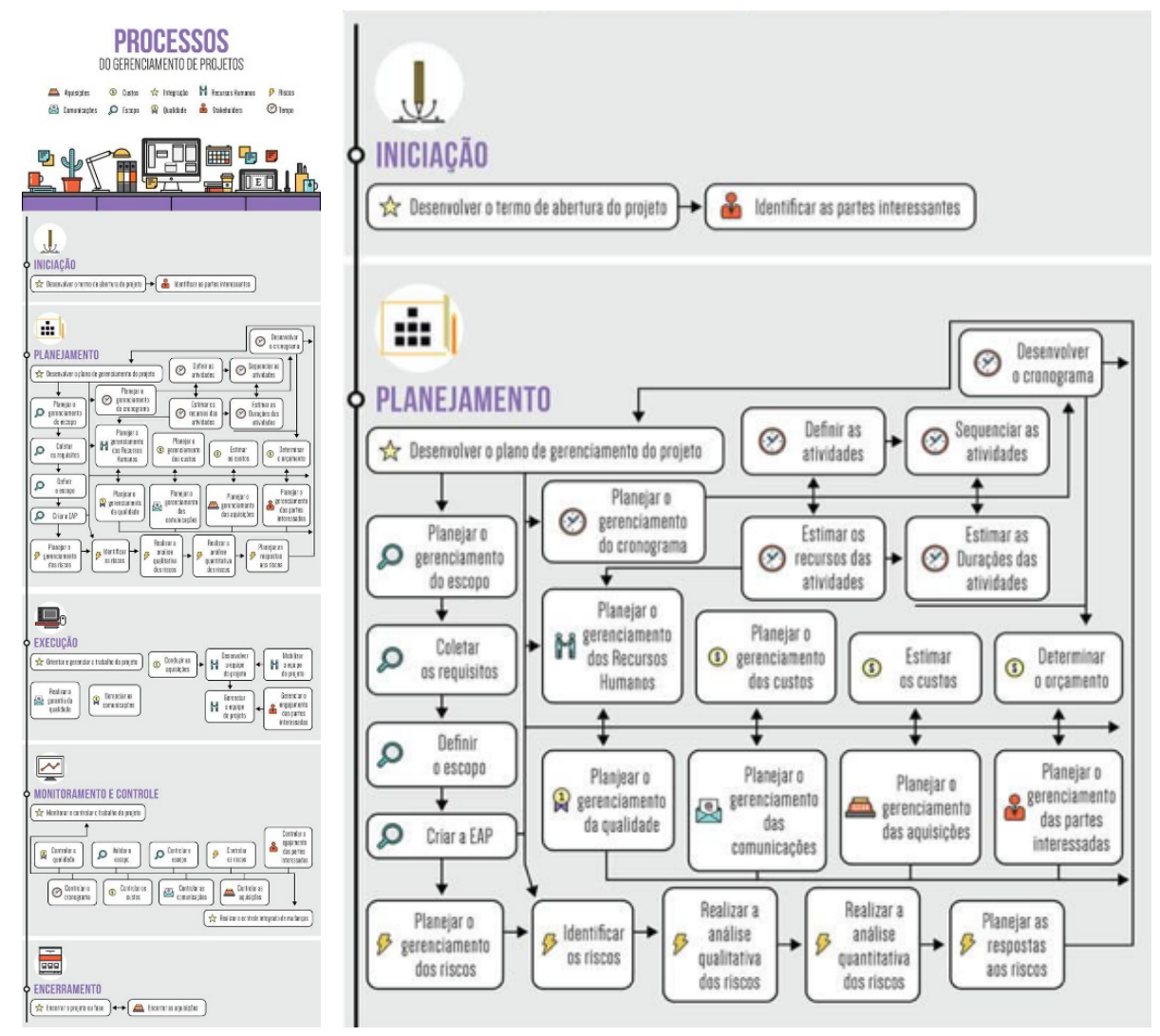

Figura 8 Representação 10 - Infográfico: Processos de gerenciamento de projetos. Fonte: Aquiles (2016). 
A análise foi realizada a partir dos parâmetros propostos por Engelhardt (2002) considerados mais relevantes para aplicação em métodos e processos. Os resultados são apresentados a seguir.

\section{Resultados da análise}

A análise possibilitou identificar tendências quanto a utilização dos elementos gráficos nas representações. No Quadro 2 são apresentadas as incidências das variáveis encontradas na análise.

Quadro 2 Síntese dos resultados da análise gráfica: Fonte: as autoras.

(Continua)

\begin{tabular}{|c|c|c|c|c|c|c|c|c|c|c|c|}
\hline \multirow{2}{*}{$\begin{array}{c}\text { Legenda: } \square \text { Contém a variável } \\
\text { Representações }\end{array}$} & \multicolumn{11}{|c|}{ Não contém a variável } \\
\hline & 1 & 2 & 3 & 4 & 5 & 6 & 7 & 8 & 9 & 10 & Total \\
\hline \multicolumn{12}{|l|}{ Atributos visuais } \\
\hline \multicolumn{12}{|l|}{ O Espaciais: } \\
\hline Forma & & & & & & & & & & & 10 \\
\hline Tamanho & & & & & & & & & & & 8 \\
\hline Orientação & & & & & & & & & & 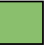 & 8 \\
\hline Posição & & & & & & & & & & & 10 \\
\hline \multicolumn{12}{|l|}{ O Preenchimento: } \\
\hline Cor & & & & & & & & & & & 10 \\
\hline Textura & & & & & & & & & & & 0 \\
\hline Valor & & & & & & & & & & & 4 \\
\hline \multicolumn{12}{|l|}{ Estruturas sintáticas básicas } \\
\hline \multicolumn{12}{|l|}{ O Relação objeto-objeto: } \\
\hline Agrupamento & & & & & & & & & & & 6 \\
\hline Separação & & & & & & & & & & & 7 \\
\hline Alinhamento & & & & & & & & & & & 8 \\
\hline Vinculação & & & & & & & & & & & 10 \\
\hline Contenção & & & & & & & & & & ] & 10 \\
\hline Sobreposição & & & & & & & & & & & 7 \\
\hline \multicolumn{12}{|l|}{ O Relação objeto-espaço: } \\
\hline Estruturas espaciais não ancoradas & & & & & & & & & 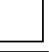 & 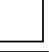 & 3 \\
\hline Estruturas espaciais não ancoradas & & & & & & & & & & & 7 \\
\hline \multicolumn{12}{|l|}{ Estruturas sintáticas compostas } \\
\hline Combinação simultânea & & & & & & & & & & & 5 \\
\hline Aninhamento & & & & & & & & & & & 8 \\
\hline Inserção de fundo & & & & & & & & & & & 7 \\
\hline
\end{tabular}


Quadro 2 Síntese dos resultados da análise gráfica: Fonte: as autoras.

\begin{tabular}{|c|c|c|c|c|c|c|c|c|c|c|c|}
\hline \multirow{2}{*}{$\begin{array}{c}\text { Legenda: } \square \text { Contém a variável } \\
\text { Representações }\end{array}$} & \multicolumn{11}{|c|}{ Não contém a variável } \\
\hline & 1 & 2 & 3 & 4 & 5 & 6 & 7 & 8 & 9 & 10 & Total \\
\hline \multicolumn{12}{|l|}{ Interpretação das representações } \\
\hline \multicolumn{12}{|l|}{ O Tipos de correspondência: } \\
\hline Literal & & & & & & & & & & & 5 \\
\hline Metafórico & & & & & & & & & & $\square$ & 5 \\
\hline \multicolumn{12}{|l|}{ O Modo de expressão: } \\
\hline Pictóricos & & & & & & & & & & & 8 \\
\hline Não pictóricos & & & & & & & & & & $\square$ & 2 \\
\hline \multicolumn{12}{|l|}{ O Significado dos objetos gráficos: } \\
\hline Informativos & & & & & & & & & & & 10 \\
\hline Referenciais & & & & & & & & & 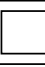 & $\square$ & 5 \\
\hline Decorativos & & & & & & & & & . & 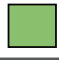 & 4 \\
\hline \multicolumn{12}{|l|}{ Classificação das representações } \\
\hline \multicolumn{12}{|l|}{ O Representações primárias: } \\
\hline Diagrama de agrupamento & & & 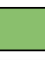 & & & & & & \begin{tabular}{|c|c|c|} 
\\
\end{tabular} & \begin{tabular}{|r} 
\\
\end{tabular} & 4 \\
\hline Diagrama de vinculação & & & & & & & & & & 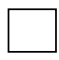 & 6 \\
\hline
\end{tabular}

Referente aos atributos visuais (espaciais e de preenchimento), observa-se a utilização de formas, cores, posição em todas representações $(n=10)$. Já a variação de orientação, tamanho e valor foram apresentadas em oito, oito e quatro representações, respectivamente. A variável textura não foi identificada. As formas usadas foram majoritariamente retângulos e círculos com função principal de container, auxiliando no agrupamentos de informações, objetos gráficos e textos (Figura 9). A cor demonstrou diversas funções, como diferenciadora dos objetos gráficos (preenchimento, contorno, linhas), demarcando etapas pela sua variação e indicação de agrupamento de informações (Figura 10). O valor, embora pouco utilizado, foi observado como diferenciador cromático no preenchimento dos objetos gráficos, sendo eficazes na indicação de grupos e subgrupos (Figura 10). A orientação definiu a direção dos objetos no espaço gráfico, e também a noção de movimento e sequencialidade das etapas e fluxo de informações, criando ordem de visualização e leitura (Figura 9). O tamanho foi utilizado para hierarquizar informações, indicando grupos e seus subgrupos, da mesma forma que a posição situou os elementos no espaço gráfico.

Quanto às estruturas sintáticas básicas (relação objeto-objeto/ objeto-espaço) seis das dez representações apresentaram a variável agrupamento, sete separação e sobreposição, oito alinhamento, 


\section{A Orientação das setas, B Forma como container}

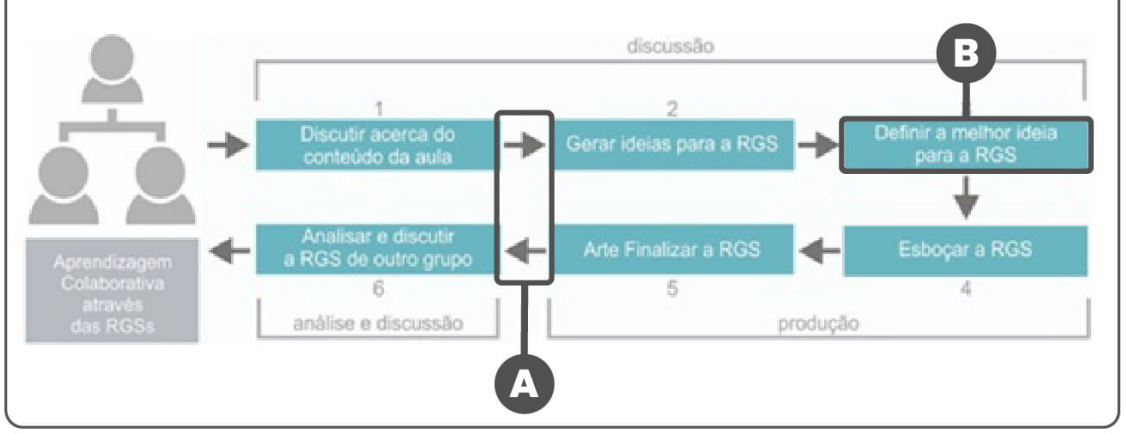

Figura 9 Exemplo de estruturas identificadas na análise - Utilização de orientação e forma.

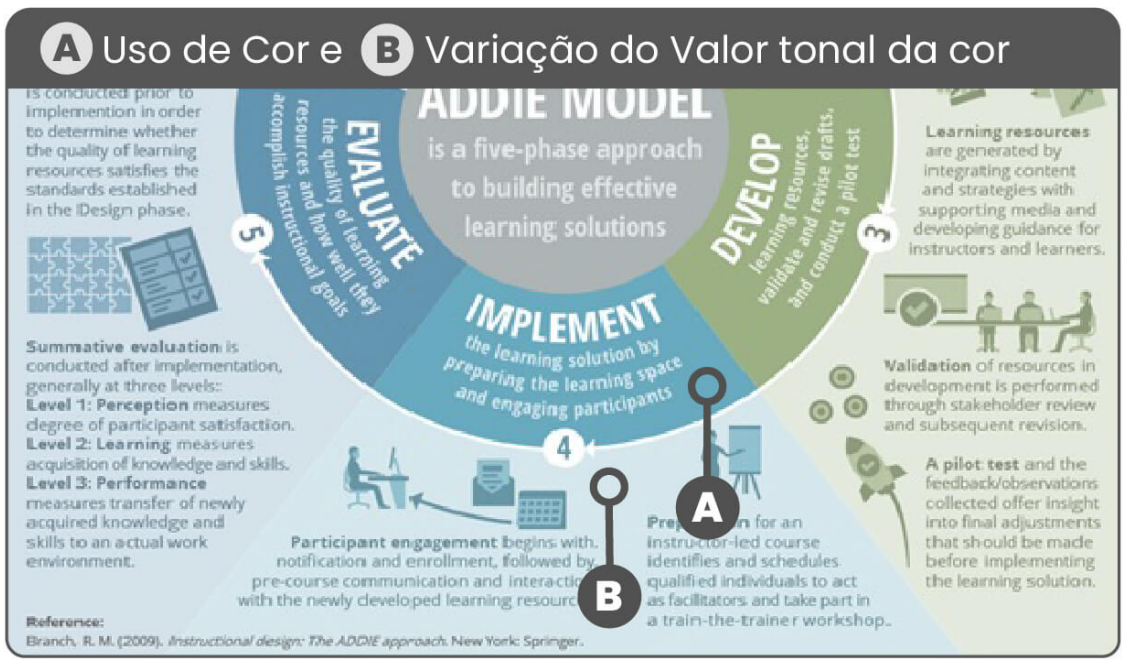

Figura 10 Exemplo de estruturas identificadas na análise - Utilização da cor e valor tonal.

todas $(n=10)$ vinculação e contenção. A separação ocorreu pelo uso de linhas localizadoras, ou espaçamentos entre blocos de informação no espaço gráfico, auxiliando na visualização e organização dos dados. As relações de alinhamento, vinculação e contenção foram recorrentes nas amostras a partir da utilização dos atributos espaciais, previamente descritos. Destaca-se aqui a vinculação ocorrida a partir de linhas localizadoras contínuas, tracejadas e pontilhadas, pontos localizadores, e setas, que geralmente ligam containers ou agrupamentos de informação. Essas auxiliam no direcionamento, ordem de leitura dos dados, e expressam movimento sendo assim, relevantes para representação de métodos e processos. A sobreposição ocorreu pela justaposição de objetos gráficos de diferentes níveis sintáticos, criando diferentes camadas de informação. A Figura 11 apresenta um exemplo de aplicação de agrupamento, separação, alinhamento, vinculação e contenção. 


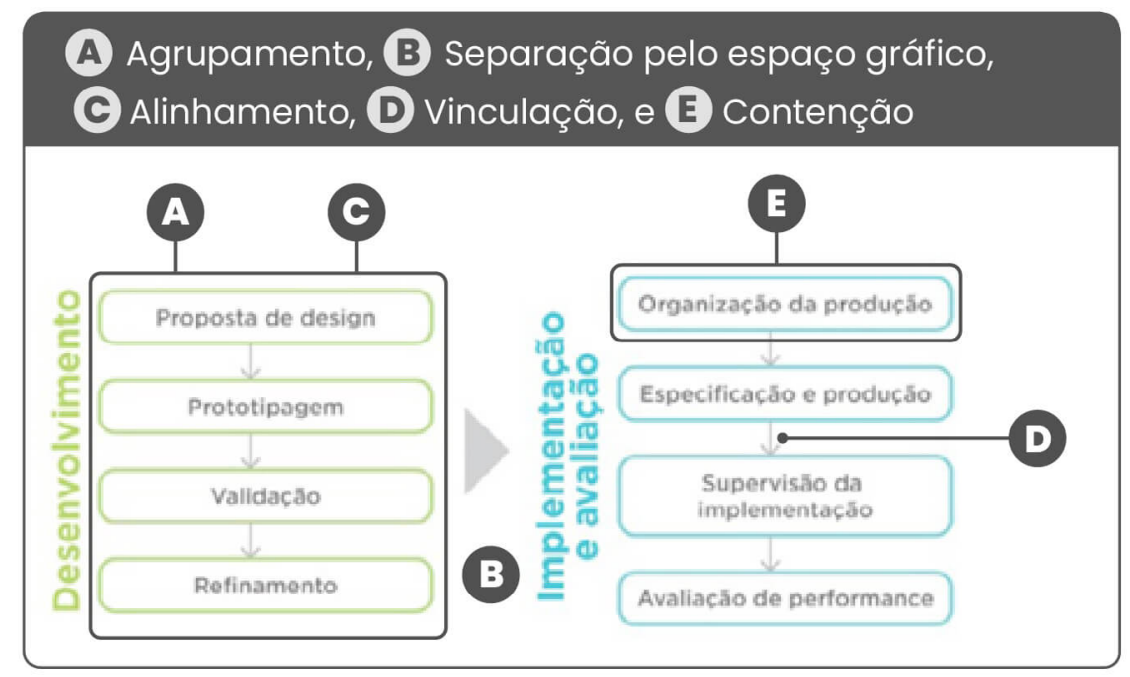

Figura 11 Exemplo de estruturas identificadas na análise - Utilização de agrupamento, separação, alinhamento, vinculação e contenção.

Quanto à relação objeto-espaço, três das dez representações, apresentaram estruturas espaciais não ancoradas, e sete ancoradas. Quando não ancoradas, as estruturas não apresentaram ligação direta dos objetos gráficos ao espaço gráfico, enquanto as ancoradas apresentaram.

Quanto às estruturas sintáticas compostas, cinco das dez representações apresentaram combinação simultânea, oito apresentaram aninhamento, e sete inserção de fundo. A combinação simultânea foi evidenciada pela incidência de objetos gráficos que participavam em mais de uma estrutura sintática em mesmo nível simultaneamente. A Figura 12 apresenta um exemplo de combinação simultânea de estruturas sintáticas.

$\mathrm{O}$ aninhamento ocorreu pelo posicionamento de um objeto gráfico composto servindo como único em um nível superior da decomposição, e a inserção de fundo através do uso de um objeto gráfico de nível inferior delimitando a superfície da representação.

Combinação simultânea de: (A) Contenção, B Separação, C Vinculação, e (D) Alinhamento em mesmo nível sintático

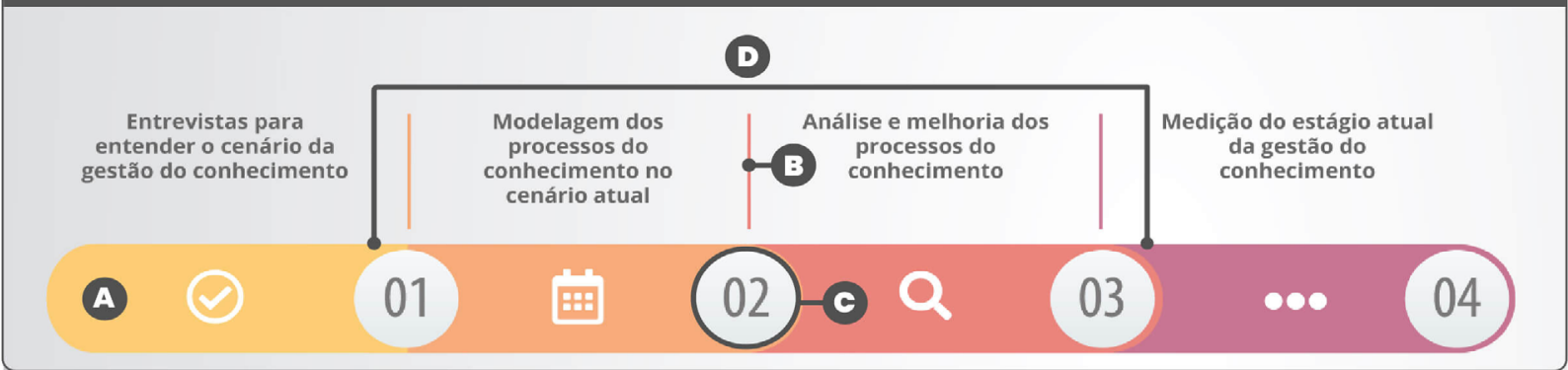

Figura 12 Exemplo de estruturas identificadas na análise - Uso de combinação simultânea de contenção, separação, vinculação e alinhamento. 
As variáveis de aninhamento e inserção de fundo são exemplificadas nas Figuras 13 e 14 .

Quanto à interpretação das representações gráficas, no referente aos tipos de correspondência cinco das dez representações apresentaram correspondência literal e cinco metafórica. A literal foi evidenciada pelo uso de estruturas sintáticas representando esquematicamente os processos e métodos, baseadas na similaridade ao processo real. Enquanto a metafórica ocorreu pela analogia entre as ilustrações e seu real significado.

Referente ao modo de expressão, oito das dez representações, apresentaram expressões pictóricas (ilustração/pictogramas), (Figura 15), e apenas duas não pictóricas (elementos esquemáticos), como exemplificado pela Figura 16. Já no significado dos objetos gráficos, todas $(\mathrm{n}=10)$ apresentaram objetos com significado informativo, cinco referenciais e quatro decorativos. Observou-se maior uso do significado informativo, sendo o foco nesse contexto a transmissão da informação e entendimento da mesma, enquanto os objetos de significado referencial foram auxiliares na visualização, e os decorativos serviram a propósitos estéticos.

Quanto às classificações das representações, quatro das dez representações classificaram-se como diagrama de agrupamento (categorização de elementos a partir de containers e separadores), e seis como diagrama de vinculação (elementos de lincagem).

Dentre as dez representações analisadas, seis apresentaram maior incidência de variáveis analisadas, sendo elas as representações: 1, 3, 4, 8, 9, e 10. Entre as representações, entendemos que cada uma possui diferentes características adequadas a seu contexto de uso e grau de
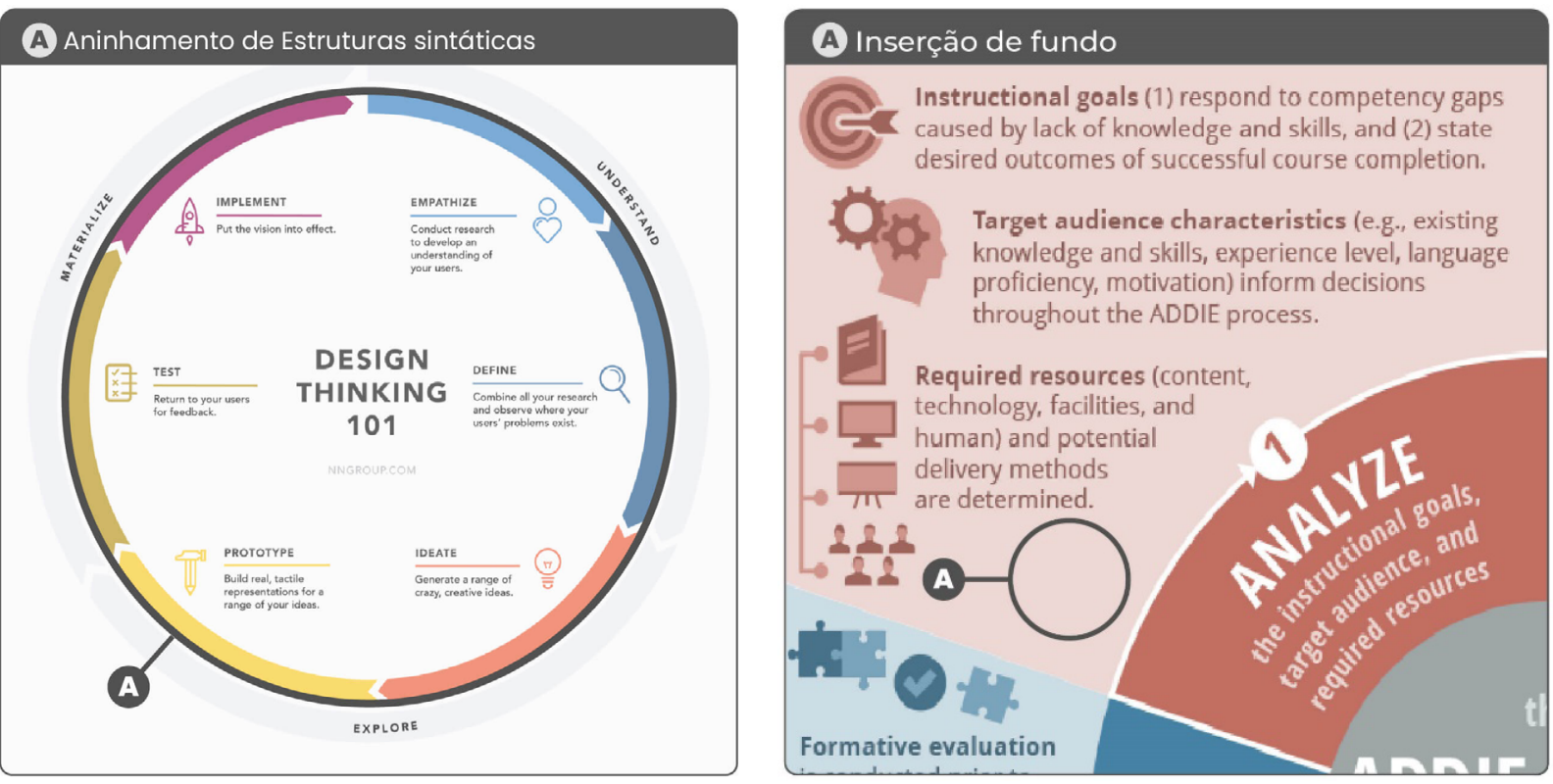

Figuras 13 e 14 Exemplo de estruturas identificadas na análise - Utilização de aninhamento e inserção de fundo. 

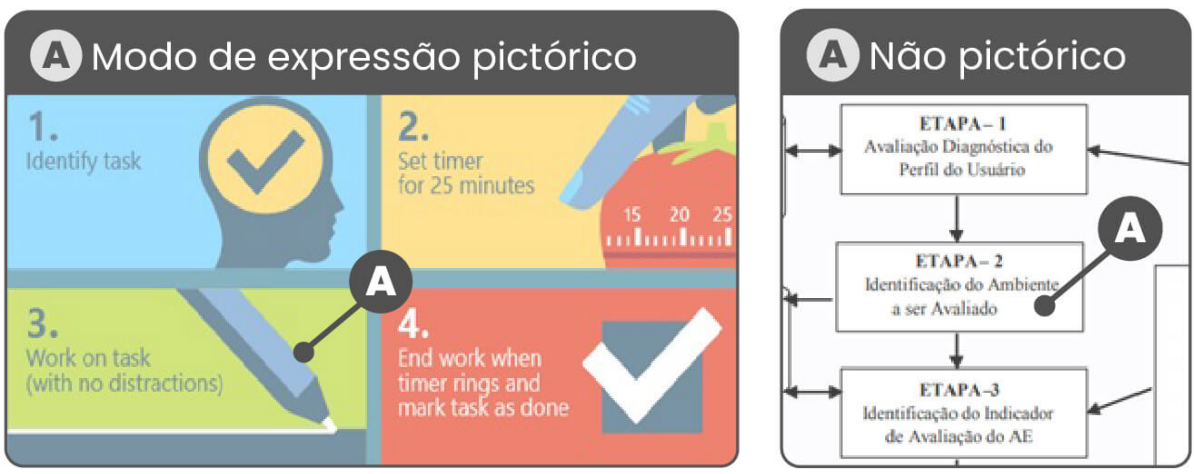

Figuras 15 e 16 Exemplo de estruturas identificadas na análise - Uso do modo de expressão pictórica pelo uso de ilustrações, e uso do modo de expressão não pictórico pelo uso de elementos esquemáticos.

complexidade. Entretanto, a representação número 9 apresentou uma maior facilidade quanto ao entendimento, junto do uso adequado de diversas variáveis. Ainda assim, existem pontos de melhoria quanto à ancoragem dos objetos textuais e sua vinculação com a etapa pertencente.

\section{Considerações finais}

A abordagem teórica e analítica deste trabalho possibilitou reconhecer o papel das variáveis gráficas utilizadas em representações de processos, de forma sintática e semântica. O estudo de cada atributo evidenciou suas possíveis funções na composição, assim como as relações estabelecidas entre objetos e espaço gráfico. O uso conjunto das variáveis visuais se faz relevante principalmente para organizar, categorizar e hierarquizar os dados. Desta forma a representação pode assumir seu papel de artefato cognitivo facilitador na compreensão, conforme Norman $(1991,1993)$ e Patterson et al. (2018), das sequências e etapas de processos.

Visando a compreensão das etapas compositivas de um método, desdobramento futuro deste estudo, são aqui considerados requisitos para representação gráfica de um método, todos atributos visuais (espaciais e de preenchimento). Estes serão indispensáveis para evidenciar relações de alinhamento, agrupamento/separação, vinculação, sobreposição, contenção, ancoragem e interpretação.

Por fim, verifica-se a importância desse estudo para a área do Design da Informação, como base para a produção de representações gráficas que visem evidenciar a compreensão de sequência de informações e relacionamentos presentes nas etapas de métodos e processos. 


\section{Referências}

AQUILES, E. Infográfico: Processos de gerenciamento de projetos. 2016. Disponível em: https://www.behance.net/gallery/36770731/Processos-doGerenciamento-de-Projetos-Infografico. Acesso em: 25 fev. 2021.

BARBOSA, F. J. M.; FRANÇA. R. S.; RODRIGUES, R. F.; PARREIRAS, F. S. Visualização da informação e métodos visuais como ferramentas estratégicas para o gerenciamento de projetos. Revista Gestão e Projetos: GeP, v. 9, n. 1, p. 102114, 2018.

BUENO, J.; PADOVANI, s. Estudo do processo de aprendizagem colaborativa através das representações gráficas de síntese (RGSS). In: CONGRESSO INTERNACIONAL DE DESIGN DA INFORMAÇÃo, 7., 2015, Brasília. Anais [...]. São Paulo: Blucher, 2015. p. 374-385.

ENGELHARDT, Y. The language of graphics: a framework for the analysis of syntax and meaning in maps, charts and diagrams. 2002. Tese. (Doutorado) Institute for Logic, Language and Computation, Universiteit van Amsterdam, Amsterdam, 2002.

GIL, A. C. Métodos e técnicas de pesquisa social. 6. ed. São Paulo: Atlas, 2008. IGTI, INSTITUTO DE GESTÃO E TECNOLOGIA DA INFORMAÇÃO. Implementando a gestão do conhecimento. 2018. Disponível em: https://www.igti.com.br/ implementando-a-gestao-do-conhecimento. Acesso em: 25 fev. 2021.

KEMCZINSKI, A. Método de avaliação para ambientes e-learning. 2005. Tese (Doutorado em Engenharia de Produção) - Programa de Pós-graduação em Engenharia de Produção, Universidade Federal de Santa Catarina, Florianópolis, 2005.

LEAN BLOG. Ferramentas de análise: diagrama de Ishikawa e gráfico de pareto. 2018. Disponível em: https://terzoni.com.br/leanblog/diagrama-ishikawa-egrafico-de-pareto/. Acesso em: 3 dez. 2020.

LIMA, F. U. Processos organizacionais. Universidade Federal do Amapá, 2017. MiCrosoft PARTNER COMmUnity. The pomodoro technique. 2017. Disponível em: https://www.microsoftpartnercommunity.com/t5/Blog-Discussions/ Work-smarter-not-harder-The-Pomodoro-Technique/m-p/2828. Acesso em: 25 fev. 2021.

NFE.io, Nota fiscal eletrônica. Como criar um workflow em 9 passos. 2017. Disponível em: https://nfe.io/blog/gestao-empresarial/sugestoes-demelhorias-para-uma-empresa/. Acesso em: 25 fev. 2021.

NORMAN, D. A. Cognitive artifacts. In: CARROLL, J. M.; LONG, J. (orgs.). Designing interaction: psychology at the human-computer interface. Reino Unido: Cambridge University Press, 1991. p. 17-38.

NORMAN, D. A. Things that make us smart: defending human attributes in the age of the machine. Cambridge: Perseus books, 1993.

OBSIDIAN LEARNING. Instructional design resource: ADDIE Infographic. 2017. Disponível em: https://www.obsidianlearning.com/blog/2017/o2/ instructional-design-resource-addie-infographic.html. Acesso em: 24 fev. 2021.

octADESK. Metodologia Octadesk: garantia de qualidade no atendimento ao cliente. 2017. Disponível em: https://blog.octadesk.com/metodologiaqualidade-no-atendimento-ao-cliente/. Acesso em: 26 fev. 2021. 
PADOVAnI, s. Representações gráficas de síntese: artefatos cognitivos no ensino de aspectos teóricos em design de interface. Revista Educação Gráfica, v. 16, n. 2, p. 123-142, 2012.

SMYthe, к. C. A. s. Proposta de método de obtenção de dados sobre comportamento informacional dos usuários no processo de wayfinding em ambientes hospitalares. 2018. Tese (Doutorado em Design) - Setor de Artes, Comunicação e Design, Universidade Federal do Paraná, Curitiba, 2018. SMYTHe, K. C. A. S.; PRADO, G. C.; SMYTHE-JR.; N. L. Análise de formas de representação gráfica dos requisitos projetuais utilizadas no processo de design de produtos assistivos. InfoDesign, v. 13, n. 1, p. 72-92, 2016.

\section{Sobre as autoras}

\section{Diovana Mazur Damacena}

diovanamazur9@gmail.com

Discente do Departamento de Design da Universidade Federal do Paraná (UFPR), Curitiba, PR

\section{Kelli C. A. Silva Smythe}

kellicas@gmail.com

Professora Adjunta do Departamento de Design da Universidade Federal do Paraná (UFPR), Curitiba, PR

Artigo recebido em/Submission date: $20 / 8 / 2021$ Artigo aprovado em/Approvement date: 2/9/2021 